\title{
TI.26.1
}

\section{Introduction to Identity Federations}

- PDF: IntroductiontoldentityFederations.pdf

- Text: IntroductiontoldentityFederations.txt

\section{More Information}

\begin{tabular}{|l|l|}
\hline Repository ID & TI.26.1 \\
\hline Persistent URL & http://doi.org/10.26869/TI.26.1 \\
\hline Title & Introduction to Identity Federations \\
\hline Authors & David Walker <0000-0003-2540-0644>, Ann West \\
\hline Sponsor & Internet2 \\
\hline Review & Consultation for Trusted Relationships for Access Management: The InCommon Model \\
\hline Status & Review \\
\hline Publish Date & $3 / 7 / 2017$ \\
\hline DOI & $10.26869 /$ TI.26.1 \\
\hline Signature & \\
\hline Deprecated & Yes \\
\hline Future Review & $12 / 1 / 2018$ \\
\hline Supersedes & \\
\hline Format & PDF \\
\hline Related Docs & TI.3.1 \\
\hline Development Location & \\
\hline IP Framework & CC BY 4.0 \\
\hline Subject Tags & policy, service \\
\hline Notes & \\
\hline
\end{tabular}

